\title{
Prevalence and associated factors for dipstick microscopic hematuria in men
}

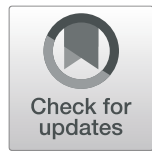

Karim Courtemanche, Peter Chan and Wassim Kassouf 'D

\begin{abstract}
Background: Microscopic hematuria is a common incidental finding on routine urinalysis. Although there are no clear recommendations to perform routine urinalysis, some studies have shown that up to $50 \%$ of general practitioners continue to perform annual routine urinalysis regardless of age or risk factors. The aim of this study was to identify associated factors and prevalence of dipstick microscopic hematuria in the general male population presenting to an annual public men's health fair.

Method: We conducted a retrospective analysis of prospectively collected data at an annual Men's Health fair from 2008 to 2013. Patient reported health questionnaires, basic physical exam including digital rectal exam, basic bloodwork and dipstick urinalysis data was examined.

Results: A total of 979 patients were reviewed. Of these, 850 provided a urine sample and were included in the final analysis. Seventy-three (8.6\%) patients had positive hematuria on urinalysis. Average age in both groups was 55 years. Presence of microscopic hematuria was correlated with presence of diabetes and proteinuria with odds-ratio of 2.8 (1.35.8) and 2.9 (1.7-5.0) respectively on multivariate analysis. There was no significant correlation identified with age, hypertension, coronary artery disease, body-mass index, smoking, prostate specific antigen (PSA) or International Prostate Symptom Score (IPSS). Limitation of this study is the lack of follow-up and knowledge of subsequent investigations of patients.

Conclusion: Microscopic hematuria is a prevalent condition in the male population presenting to a health fair. The only factors associated with microscopic hematuria were diabetes and proteinuria. No association was found between hematuria and smoking, age, or lower urinary tract symptoms.
\end{abstract}

Keywords: Microscopic hematuria, Prevalence, Risk factors

\section{Background}

Many etiologies exist for microscopic hematuria such as infection, urolithiasis, benign prostate hyperplasia, malignancy, nephropathies or physiologic. About $3.6 \%$ of patients presenting with microscopic hematuria will present a urinary tract malignancy [1] but positive predictive value of microhematuria for malignancy is low and thus population screening has not been recommended [1-3]. The definition of microscopic hematuria is three or greater red-blood cells (RBC) per high power field on a properly collected microscopic urinalysis as per the American Urological Association guidelines [1] whereas the Canadian Urological Association guidelines require confirmation with a second microscopic urinalysis

\footnotetext{
* Correspondence: wassim.kassouf@muhc.mcgill.ca

Division of Urology, McGill University Health Center, 1001 Blvd Decarie, room E02.4366, Montreal, Quebec H4A 3J1, Canada
}

[4]. However, in clinical practice, microscopy is often not ordered or not performed by the laboratory. Several studies have confirmed that dipstick urinalysis has good specificity (65-95\%) and sensitivity (91-100\%) in detecting microhematuria; [5] rates of false positives and negatives are low and can be due to hemoglobinuria, myoglobinuria, ascorbic acid or reducing agents such as povidone.

Routine screening dipstick urinalysis is currently not recommended by any organization, including the Canadian Task Force on Preventive Health Care, as there is insufficient data to support this type of screening [6]. However, up to $50 \%$ of general practitioners perform a routine urinalysis on all their patients as part of annual health screening visits [7]. Microscopic hematuria $(\mathrm{MH})$ is a common finding during routine urinalysis and leads to frequent referrals to urologists and nephrologists [8]. According to

(c) The Author(s). 2019 Open Access This article is distributed under the terms of the Creative Commons Attribution 4.0 International License (http://creativecommons.org/licenses/by/4.0/), which permits unrestricted use, distribution, and 
current guidelines, $\mathrm{MH}$ often prompts a workup of the lower and upper tracts with cystoscopy and abdominal imaging representing a significant cost to health care systems [4].

Our primary objective was to identify and describe factors associated with microscopic hematuria. We also report on the prevalence of $\mathrm{MH}$ in the male population presenting to a men's health fair.

\section{Methods}

Men who presented to an annual McGill University public men's health fair held in public venues in Montreal, Canada from 2008 to 2013 were included. Informed consents were obtained from all participants to permit anonymous analyses of collected data for educational and academic research purposes. All men who presented to this annual health fair were eligible for inclusion into the study. In addition to physical examination, patients were asked to complete a health questionnaire including socio-demographic data, medical history, medications, lifestyle (smoking, alcohol use and drugs), Metabolic Equivalent of Task scale (METs), Sexual Health Inventory for Men (SHIM), Androgen Deficiency in Aging Male (ADAM), Berlin questionnaire for obstructive sleep apnea (BQ), International Prostate Symptom Score (IPSS) and Overactive Bladder 8 (OAB8). Depending on their age, patients were offered prostate specific antigen (PSA), cholesterol panel and testosterone level testing. They also underwent a digital rectal exam and a dipstick urinalysis.

Patients who stated a previous history of hematuria or did not provide a urine sample were excluded from the final study population. Baseline characteristics were compared using t-tests for continuous variables and chi-square or Fisher exact for categorical variables depending on the number of patients in that particular analysis. Multivariate logistic regression was used to examine various factors and their association with microscopic hematuria and establish the odds-ratio of microhematuria on dipstick urinalysis. Where data was missing, analysis was done only with patients with complete records available. Missing data was primarily due to patients not answering specific questions on the questionnaire. Significant $p$ values were set as twotailed $p<0.05$. Data was analyzed using SPSS version 17 .

\section{Results}

Data was reviewed on 979 patients who presented to the men's health fair from 2008 to 2013; 850 provided a urine sample and had no history of previous hematuria and formed the basis of this report. Mean and median age of men was 54.7 and 55 respectively (range 20 to 87). Baseline demographics of the population are presented in Table 1 Mean body-mass index (BMI) was 27.6. Of 850 patients, 67 (7.9\%) had diabetes mellitus
Table 1 Demographics and comorbidities

\begin{tabular}{ll}
\hline Age in year (range) & 55 (20-87) \\
\hline Education Level & Grade 7 or lower: 3.1\% (26) \\
& Some high school: 8.9\% (76) \\
& High school graduate or CEGEP: 24.8\% (211) \\
& Some University: 13.1\% (111) \\
& University Graduate: 37.2\% (316) \\
& Post-graduate studies: 11.4\% (97) \\
& Non-smoker: 67.9\% (573) \\
& Smoker: $12.3 \%$ (104) \\
Smoking history & Ex-smoker: 19.8\% (167) \\
& $27.6 \pm 5.0$ \\
BMI (kg/m $\left.{ }^{2}\right)$ & Overall: 73 (8.6\%) \\
Dipstick hematuria & $+: 41(4.8 \%)$ \\
& $++: 17(2 \%)$ \\
& $+++: 11(1.3 \%)$ \\
Proteinuria & $++++: 4(0.5 \%)$ \\
Diabetes & $128(15.1 \%)$ \\
Hypertension & 67 (7.9\%) \\
Coronary artery disease & $156(18.4 \%)$ \\
& $46(5.4 \%)$ \\
& $1.54 \pm 1.74$ \\
\hline
\end{tabular}

(DM), 46 (5.4\%) had coronary artery disease (CAD), 156 (18.4\%) had hypertension (HTN) and 18 (2.1\%) had a history of malignancy. From a urological aspect, 42 (4.9\%) had a history of previous urological surgery, 42 (4.9\%) had a history of urinary or urological infections and $41(4.8 \%)$ had a history of stones.

Seventy-three (8.6\%) patients demonstrated MH. There was no significant difference in rates of coronary artery disease, previous history of urolithiasis, malignancy, urological surgery, urological infections or hypertension between patients with and without MH. Smoking rates (present, past, ever) were similar in both groups (Table 2). Distribution of IPSS score categories was similar between both groups ( $6.1 \%$ vs $6.3 \%$ severe scores, $p=0.825)$. There was no difference between SHIM score categories ( $p=$ $0.479)$. On multivariable analysis, the only factors associated with $\mathrm{MH}$ were diabetes (OR 2.8, $p=0.007$ ) and proteinuria (OR 2.9, $p=0.0001$ ) (Table 3).

Discussion This study demonstrated that microscopic hematuria is prevalent in the male population and diabetes is the primary factor associated with increased risk of detecting $\mathrm{MH}$ on dipstick urinalysis.

Multiple studies evaluated the prevalence of microscopic hematuria with varying rates in a general screening population. Our observed prevalence of microscopic hematuria was $8.6 \%$ which is similar to other studies in 
Table 2 Comparison of associated factors

\begin{tabular}{|c|c|c|c|c|}
\hline Characteristic & No hematuria $(n=777)$ & Microhematuria $(n=73)$ & $N$ & $\mathrm{p}$ \\
\hline Age & $54.7(+/-12.9)$ & $55.4(+/-10.6)$ & 850 & 0.669 \\
\hline Diabetes mellitus & $7.1 \%(55)$ & $16.4 \%(12)$ & 850 & 0.005 \\
\hline Hypertension & $18.3 \%(142)$ & $19.2 \%(14)$ & 850 & 0.849 \\
\hline Coronary artery disease & $5.4 \%(42)$ & $5.5 \%(4)$ & 850 & 1.000 \\
\hline Malignancy & $2.2 \%(17)$ & $1.4 \%(1)$ & 850 & 1.000 \\
\hline Urological surgery & $4.8 \%(37)$ & $6.8 \%(5)$ & 850 & 0.431 \\
\hline History of urolithiasis & $4.8 \%(37)$ & $5.5 \%(4)$ & 850 & 0.773 \\
\hline Urological infections & $5.1 \%(40)$ & $2.7 \%(2)$ & 850 & 0.571 \\
\hline Smoking & $12.4 \%(96)$ & $11.3 \%(8)$ & 844 & 0.778 \\
\hline Proteinuria & $13.4 \%(104)$ & $32.9 \%(24)$ & 850 & 0.0001 \\
\hline \multirow[t]{3}{*}{ IPSS category (mild, mod, severe) } & Mild: 58\% (407) & Mild: 61.9\% (39) & 765 & 0.825 \\
\hline & Moderate: 35.9\% (252) & Moderate: 31.7\% (20) & & \\
\hline & Severe: $6.1 \%(43)$ & Severe: $6.3 \%$ (4) & & \\
\hline OAB-q v8 & $39.6 \%(280)$ & $34.3 \%(23)$ & 774 & 0.434 \\
\hline \multirow[t]{5}{*}{ SHIM } & No ED: $48 \%$ (306) & No ED: $53.2 \%(33)$ & 797 & 0.479 \\
\hline & Mild: $28.7 \%$ (183) & Mild: $27.4 \%(17)$ & & \\
\hline & Mild-Mod: 3.3\% (85) & Mild-Mod: 8.1\% (5) & & \\
\hline & Moderate: 6.1\% (39) & Moderate: 9.7\% (6) & & \\
\hline & Severe: $3.9 \%(25)$ & Severe: $1.6 \%(1)$ & & \\
\hline ADAM questionnaire positive & $64.4 \%(482)$ & $52.9 \%(37)$ & 819 & 0.069 \\
\hline Metabolic syndrome & $28.5 \%(142)$ & $39.4 \%(13)$ & 532 & 0.234 \\
\hline $\mathrm{PSA}>4 \mathrm{ng} / \mathrm{ml}$ & $7.3 \%(47)$ & $7.5 \%(5)$ & 715 & 0.950 \\
\hline \multirow[t]{3}{*}{ BMI } & $<25: 28.8 \%(220)$ & $<25: 26.0 \%(19)$ & 837 & 0.688 \\
\hline & 25-30: 45.5\% (348) & $25-30: 43.8 \%(32)$ & & \\
\hline & > 30: 25.7\% (196) & > 30: $30.1 \%(22)$ & & \\
\hline
\end{tabular}

men ranging from $2.5 \%$ [9] to 13\% [10]. Previous studies have examined the risk factors of urinary tract malignancy diagnosed following workup of hematuria. Risk factors of malignancy identified in some of these studies were age, smoking, the presence of gross hematuria and positive cytology [11-13]. Kang et al. found in a screening study that male sex and diabetes increased the risk of finding underlying pathology on workup of MH [12]. None of the

Table 3 Multivariate logistic regression analysis of factors associated with microhematuria

\begin{tabular}{lll}
\hline Variable & OR & $p$ \\
\hline Diabetes & $2.758(1.312-5.799)$ & 0.007 \\
Hypertension & $0.775(0.383-1.568)$ & 0.478 \\
Coronary artery disease & $0.965(0.318-2.932)$ & 0.951 \\
Smoking & $0.934(0.427-2.045)$ & 0.864 \\
Proteinuria & $2.882(1.669-4.976)$ & 0.0001 \\
BMI $\geq 30$ & $1.015(0.966-1.068)$ & 0.551 \\
Age & $0.998(0.977-1.020)$ & 0.857 \\
\hline
\end{tabular}

studies identified in our review of the literature analyzed factors associated with likelihood of detecting $\mathrm{MH}$. In our study, a past medical history of diabetes increased the odds of hematuria by 2.8 times. Okada et al. showed that microscopic hematuria had little clinical significance in diabetic nephropathy and that creatinine doubling time or increase of serum creatinine was no different between patients with persistent $\mathrm{MH}$ or without $\mathrm{MH}$ [14]. Hematuria was also significantly associated with the presence of concomitant proteinuria with an odds ratio of 2.9, likely associated with the presence of diabetes. The increase risk of finding $\mathrm{MH}$ in diabetic patients especially in the context of proteinuria could be used to better counsel patients when these abnormal results are found.

How would the identification of factors associated with $\mathrm{MH}$ help counselling the patient? The yield of finding malignancy in patients with $\mathrm{MH}$ is very low. AUA metaanalysis found that the incidence of malignancy upon workup of asymptomatic $\mathrm{MH}$ was $3.3 \%$ when based on one positive urine sample [1]. According to current guidelines, $\mathrm{MH}$ prompts a workup of the lower and 
upper tracts with cystoscopy and abdominal imaging representing a significant cost to health care systems. [4] Further prospective studies are needed to evaluate whether the identification of factors associated with increased risk of $\mathrm{MH}$ may help stratifying patients who can obviate investigations with invasive procedures.

Some limitations exist in our study. All past medical history was self-reported by patients and may thus induce a bias. The population presenting to this health fair may not be representative of the general population as patients with specific concerns or who do not have regular medical follow-up may have been overrepresented. However, the prevalence of smoking and comorbidities such as hypertension, diabetes, and coronary artery disease was similar to rates in the general Canadian population $[15,16]$. Furthermore, this was a single institution experience and this may limit generalizability of our findings. Another limitation is that the dipstick microhematuria was not confirmed with microscopy in this study. Several studies have confirmed that dipstick urinalysis has good specificity (65-95\%) and sensitivity (91-100\%) in detecting microhematuria $[5,17]$ but should still be confirmed with microscopy before ordering costly and invasive testing. Lastly and importantly, follow-up data was not available for our patient population to identify if these patients ultimately underwent full workup and their findings.

\section{Conclusion}

The prevalence of microhematuria in the general male population presenting to a public health fair was $8.6 \%$, in keeping with other reported studies of prevalence of $\mathrm{MH}$. Significant factors associated with $\mathrm{MH}$ were a history of diabetes and concomitant proteinuria.

\section{Abbreviations}

ADAM: Androgen Deficiency in Aging Male; BMl: Body-mass index; BQ: Berlin questionnaire; CAD: Coronary artery disease; DM: Diabetes mellitus; HTN: hypertension; IPSS: International prostate symptom score; METs: Metabolic equivalent of task scale; $\mathrm{MH}$ : Microscopic hematuria; OAB8: Overactive bladder 8; PSA: Prostate specific antigen; RBC: Red-blood cells; SHIM: Sexual Health Inventory for Men

\section{Acknowledgements}

Not applicable.

\section{Authors' contributions}

KC did the data entry, statistical analysis and interpretation of the data and was a major contributor in writing the manuscript. PC coordinated the collection of data from patients and contributed and approved the final manuscript. WK participated in the design of the study, reviewed the results and was a major contributor to writing the manuscript. All authors read and approved the final manuscript.

\section{Funding}

There was no funding provided for this study.

\section{Ethics approval and consent to participate}

Informed written consents were obtained from all participants to permit anonymous analyses of collected data for educational and quality of care purposes. Ethics approval was waived by the IRB of the McGill University Health Center as it was considered an annual educational and quality of care event.

\section{Consent for publication}

The results presented in this paper have not been published previously in whole or part, except in abstract form. Abstract was presented at the Canadian Urological Association annual meeting in 2015

Not applicable.

\section{Competing interests}

The authors declare that they have no competing interests. Wassim Kassouf is an associate editor for BMC Urology.

Received: 9 August 2018 Accepted: 31 July 2019

Published online: 06 August 2019

\section{References}

1. Davis R, Jones JS, Barocas DA, Castle EP, Lang EK, Leveillee RJ, Messing EM, Miller SD, Peterson AC, Turk TM, et al. Diagnosis, evaluation and follow-up of asymptomatic microhematuria (AMH) in adults: AUA guideline. J Urol. 2012;188(6 Suppl):2473-81.

2. Woolhandler S, Pels RJ, Bor DH, Himmelstein DU, Lawrence RS. Dipstick urinalysis screening of asymptomatic adults for urinary tract disorders. I. Hematuria and proteinuria. Jama. 1989;262(9):1214-9.

3. Hiatt RA, Ordonez JD. Dipstick urinalysis screening, asymptomatic microhematuria, and subsequent urological cancers in a population-based sample. Cancer Epidemiol Biomarkers Prev. 1994;3(5):439-43.

4. Wollin T, Laroche B, Psooy K. Canadian guidelines for the management of asymptomatic microscopic hematuria in adults. Can Urol Assoc J. 2009;3(1):77-80.

5. Thaller TR, Wang LP. Evaluation of asymptomatic microscopic hematuria in adults. Am Fam Physician. 1999:60(4):1143-1152, 1154

6. Krogsboll LT. Guidelines for screening with urinary dipsticks differ substantially--a systematic review. Danish Med J. 2014;61(2):A4781.

7. Yafi FA, Aprikian AG, Tanguay S, Kassouf W. Patients with microscopic and gross hematuria: practice and referral patterns among primary care physicians in a universal health care system. Can Urol Assoc J. 2011;5(2):97-101.

8. Rao PK, Gao T, Pohl M, Jones JS. Dipstick pseudohematuria: unnecessary consultation and evaluation. J Urol. 2010;183(2):560-4.

9. Ritchie CD, Bevan EA, Collier SJ. Importance of occult haematuria found at screening. Br Med J (Clin Res Ed). 1986;292(6521):681-3.

10. Britton JP, Dowell AC, Whelan P. Dipstick haematuria and bladder cancer in men over 60: results of a community study. BMJ. 1989;299(6706):1010-2.

11. Cha EK, Tirsar LA, Schwentner C, Hennenlotter J, Christos PJ, Stenzl A, Mian C, Martini T, Pycha A, Shariat SF, et al. Accurate risk assessment of patients with asymptomatic hematuria for the presence of bladder cancer. World J Urol. 2012;30(6):847-52.

12. Kang M, Lee $S$, Jeong SJ, Hong SK, Byun SS, Lee SE, Jeong CW. Characteristics and significant predictors of detecting underlying diseases in adults with asymptomatic microscopic hematuria: a large case series of a Korean population. Int J Urol. 2015;22(4):389-93.

13. Loo RK, Lieberman SF, Slezak JM, Landa HM, Mariani AJ, Nicolaisen G, Aspera AM, Jacobsen SJ. Stratifying risk of urinary tract malignant tumors in patients with asymptomatic microscopic hematuria. Mayo Clin Proc. 2013;88(2):129-38.

14. Okada T, Nagao T, Matsumoto H, Nagaoka Y, Wada T, Nakao T. Clinical significance of microscopic haematuria in diabetic nephropathy in type 2 diabetes patients with overt proteinuria. Nephrol. 2013;18(8):563-8.

15. 2009 Tracking Heart Disease and Storke in Canada. 2016. http://www.phacaspc.gc.ca/publicat/2009/cvd-avc/pdf/cvd-avs-2009-eng.pdf.

16. Robitaille C, Dai S, Waters C, Loukine L, Bancej C, Quach S, Ellison J, Campbell N, Tu K, Reimer K, et al. Diagnosed hypertension in Canada: incidence, prevalence and associated mortality. CMAJ. 2012;184(1):E49-56.

17. Matulewicz RS, DeLancey JO, Pavey E, Schaeffer EM, Popescu O, Meeks JJ. Dipstick urinalysis as a test for microhematuria and occult bladder Cancer. Bladder Cancer. 2017;3(1):45-9.

\section{Publisher's Note}

Springer Nature remains neutral with regard to jurisdictional claims in published maps and institutional affiliations. 